\title{
Chiral quantum optics in photonic sawtooth lattices
}

\author{
Eduardo Sánchez-Burillo $\odot,{ }^{1}$ Chao Wan, ${ }^{1,2}$ David Zueco, ${ }^{3,4}$ and Alejandro González-Tudela ${ }^{5}$ \\ ${ }^{1}$ Max-Planck-Institut für Quantenoptik, Hans-Kopfermann-Str. 1, 85748 Garching, Germany \\ ${ }^{2}$ Fakultät Physik at Ludwig-Maximilians-Universität, Schellingstraße 4, 80799 Munich, Germany \\ ${ }^{3}$ Instituto de Ciencia de Materiales de Aragón and Departamento de Física de la Materia Condensada, \\ CSIC-Universidad de Zaragoza, Calle Pedro Cerbuna 12, 50009 Zaragoza, Spain \\ ${ }^{4}$ Fundación ARAID, Paseo María Agustín 36, 50004 Zaragoza, Spain \\ ${ }^{5}$ Instituto de Física Fundamental IFF-CSIC, Calle Serrano 113b, Madrid 28006, Spain
}

(Received 4 July 2019; accepted 2 March 2020; published 2 April 2020)

\begin{abstract}
Chiral quantum optics has become a burgeoning field due to its potential applications in quantum networks or quantum simulation of many-body physics. Current implementations are based on the interplay between local polarization and propagation direction of light in nanophotonic structures. In this manuscript, we propose an alternative platform based on coupling quantum emitters to a photonic sawtooth lattice, a one-dimensional model with an effective flux per plaquette introduced by complex tunnelings. We study the dynamics emerging from such structured photonic bath and find the conditions to obtain quasiperfect directional emission when the emitters are resonant with the band. In addition, we find that the photons in this bath can also mediate complex emitter-emitter interactions tunable in range and phase when the emitters transition frequencies lie within a band gap. Since these effects do not rely on polarization, we propose an implementation based on circuit QED to observe this physics.
\end{abstract}

DOI: 10.1103/PhysRevResearch.2.023003

\section{INTRODUCTION}

Designing nonreciprocal optical devices at the classical and quantum level has remained a challenge for many years (see, e.g., Refs. [1-4], and references therein). On the classical level, the search was mainly focused on systems breaking Lorentz reciprocity such as magneto-optical materials. On the quantum side nanophotonic systems have emerged as a powerful candidate due to the link between the polarization and propagation direction that appears thanks to the subwavelength-light confinement [5,6]. Using this connection and the intrinsic polarization of classical and quantum emitters (QEs) many experiments have shown chiral light-matter coupling in waveguides [7-12], and harnessed it to achieve, e.g., optical isolation at the single-photon level [2]. Chiral quantum optical systems [13] have also been proposed to build spin-photon interfaces [14] and directional amplifiers $[15,16]$ in quantum networks, to engineer many-body spin or photonic states [17-21], to emit nonclassical light [22,23], or to induce exotic self-organization patterns [24], among other phenomena.

These exciting predictions have triggered a race to observe this nonreciprocal penomena also beyond the optical regime. For example, linear optical circulators have been designed in

Published by the American Physical Society under the terms of the Creative Commons Attribution 4.0 International license. Further distribution of this work must maintain attribution to the author(s) and the published article's title, journal citation, and DOI. Open access publication funded by the Max Planck Society. the microwave regime [25-28] and nonreciprocal transmission of photons has been achieved using two qubits [29]. Regarding the possibility of obtaining chiral light-matter interactions, there are already theoretical proposals to obtain them based on using complex nonlocal light-matter interactions [17-19] or with moving atoms [30], but their experimental realization remains elusive. We explore here an alternative where QEs couple locally to a photonic bath without timereserval symmetry. In particular, we use the so-called photonic sawtooth lattice [see Fig. 1(a)], also labeled as $\Delta$ chain [31] or triangle lattice $[32,33]$. This is a one-dimensional model with closed loops, which allows for complex couplings $(\phi \neq 0$ in Fig. 1) between the bosonic degrees of freedom defining an effective magnetic flux per loop [34]. We predict that one can obtain quasiperfect directional emission [13] when the QEs are resonant with the bands of the system. Rather than selecting a momentum using the destructive interference induced by nonlocal couplings [17-19] or breaking the symmetry by means of an external laser in a phononic system [35], it is the asymmetric nature of the band structure the one responsible of the chirality. The properties of the bath lead to other phenomena such as the emergence of a sublattice-dependent directional emission or QE interactions tunable in range and phase when their frequencies lie in a band gap.

The text is organized as follows. We first introduce the model in Sec. II. We then describe the single-qubit dynamics when the emitter is on resonance with the photonic band in Sec. III, characterizing the emergence of directional emission. We consider the complementary situation in Sec. IV, when the QEs are off resonance with the band (i.e., in a band gap), and we characterize both the single-QE bound states and the effective qubit-qubit interactions mediated by the 

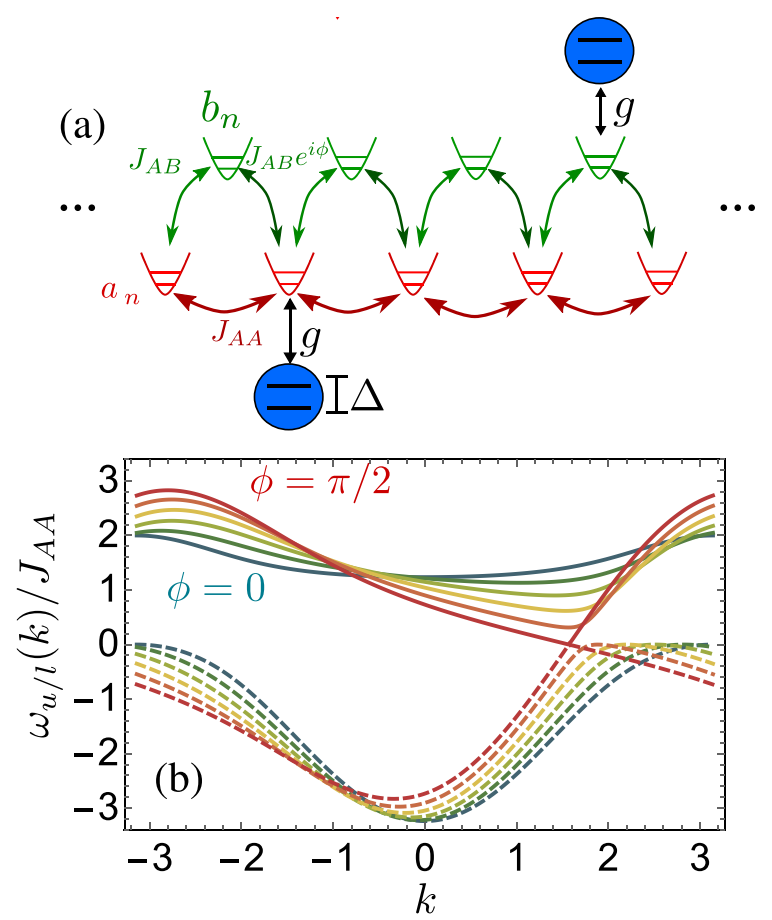

FIG. 1. (a) Two QEs (in blue), with energy gap $\Delta$, coupled with strength $g$ to a sawtooth lattice described by a bipartite lattice of coupled resonators. The red/green lattice sites denote the A/B sublattices with couplings $J_{A A}, J_{A B}$, and $J_{A B} e^{i \phi}$. (b) Bands $\omega_{u / l}(k)$ (solid/dashed) for $J_{A A}=J_{A B}$, for six equally spaced $\phi$ from $\phi=0$ (dark green) to $\phi$ and $\phi=\pi / 2$ (red).

bath. A realistic implementation for the system based on superconducting technologies is presented in Sec. V. We end up with the conclusions in Sec. VI. We leave some technical aspects for the Appendices.

\section{MODEL}

The global Hamiltonian of the system composed by the QE's and the photonic bath reads $(\hbar=1)$

$$
H=H_{\mathrm{ST}}+\Delta \sum_{j=1}^{M} \sigma_{j}^{+} \sigma_{j}^{-}+H_{\mathrm{int}},
$$

where $\Delta$ is the frequency of each $\mathrm{QE}$ described as a two-level system with ladder operators $\sigma_{j}^{+/-}$for the $j$ th $\mathrm{QE}, M$ is the number of QEs, $H_{\text {int }}$ describes the interaction, and $H_{\mathrm{ST}}$ is the Hamiltonian of the sawtooth lattice. The latter reads

$$
\begin{aligned}
H_{\mathrm{ST}}= & \omega_{B} \sum_{n=1}^{N}\left(a_{n}^{\dagger} a_{n}+b_{n}^{\dagger} b_{n}\right)-J_{A A} \sum_{n=1}^{N}\left(a_{n}^{\dagger} a_{n+1}+\text { H.c. }\right) \\
& -J_{A B} \sum_{n=1}^{N}\left(a_{n}^{\dagger} b_{n}+e^{-i \phi} a_{n+1}^{\dagger} b_{n}+\text { H.c. }\right),
\end{aligned}
$$

being $a_{n}$ and $b_{n}$ the annihilation operators of the $n$th $a$ and $b$ modes [the photonic bath is bipartite and is described by two sublattices $A$ and $B$; see Fig. 1(a)], $N$ the number of modes per sublattice, $\omega_{B}$ the energy of each resonator, that we take as the energy reference: $\omega_{B} \equiv 0, J_{A A}$ the coupling between the
$A$ sites, $J_{A B}$ the hopping strength between the $a$ and $b$ modes, and $\phi$ the phase difference in each loop.

For the interaction term $H_{\text {int }}$ [last term of Eq. (1)], we consider pointlike and dipole-field coupling under the rotatingwave approximation, valid when the coupling strength is small with respect to the other energy scales of the system [36]:

$$
H_{\mathrm{int}}=g\left(\sum_{j=1}^{M_{a}} \sigma_{j}^{+} a_{n_{j}}+\sum_{j=M_{a}+1}^{M} \sigma_{j}^{+} b_{n_{j}}\right)+\text { H.c. }
$$

Here $g$ is the coupling constant, $M_{a}$ is the number of qubits coupled to $A$ (so $M_{b} \equiv M-M_{a}$ are coupled to $B$ ), and $n_{j}$ is the position of the $j$ th qubit.

Since we are interested in predictions in the thermodynamic limit, $N \rightarrow \infty$, we take periodic boundary conditions for the bath and introduce plane-wave modes $\hat{a}_{k} / \hat{b}_{k} \equiv$ $1 / \sqrt{N} \sum_{n=1}^{N} e^{-i k n} a_{n} / b_{n}$, in terms of which $H_{\mathrm{ST}}$ [Eq. (2)] reads

$$
H_{\mathrm{ST}}=\sum_{k}\left(\hat{a}_{k}^{\dagger} \hat{b}_{k}^{\dagger}\right) h_{\mathrm{ST}}(k)\left(\begin{array}{l}
\hat{a}_{k} \\
\hat{b}_{k}
\end{array}\right) \text {, }
$$

with $h_{\mathrm{ST}}(k)$

$$
h_{\mathrm{ST}}(k)=\left(\begin{array}{cc}
-2 J_{A A} \cos k & f(k, \phi) \\
f^{*}(k, \phi) & 0
\end{array}\right),
$$

and $f(k, \phi)=-J_{A B}\left(1+e^{-i(k+\phi)}\right)$. We introduce the $\hat{\imath}$ notation to distinguish the operators in real/momentum space. We diagonalize $h_{\mathrm{ST}}(k)$ such that $H_{\mathrm{ST}}=\sum_{k}\left[\omega_{u}(k) \hat{u}_{k}^{\dagger} \hat{u}_{k}+\right.$ $\left.\omega_{l}(k) \hat{l}_{k}^{\dagger} \hat{l}_{k}\right]$, where $\hat{u}_{k}$ and $\hat{l}_{k}$ are related to $\hat{a}_{k}$ and $\hat{b}_{k}$ by means of a unitary:

$$
\left(\begin{array}{c}
\hat{u}_{k} \\
\hat{l}_{k}
\end{array}\right)=\left(\begin{array}{cc}
\cos \left(\theta_{k}\right) e^{-i \varphi_{k}} & -\sin \left(\theta_{k}\right) \\
\sin \left(\theta_{k}\right) e^{-i \varphi_{k}} & \cos \left(\theta_{k}\right)
\end{array}\right)\left(\begin{array}{l}
\hat{a}_{k} \\
\hat{b}_{k}
\end{array}\right),
$$

We provide the explicit expressions of $\theta_{k}$ and $\varphi_{k}$ in Appendix A. The bands $\omega_{u / l}(k)$ read

$$
\omega_{u / l}(k)=-J_{A A} \cos k \pm \sqrt{J_{A A}^{2} \cos ^{2} k+4 J_{A B}^{2} \cos ^{2}[(k+\phi) / 2]} .
$$

A celebrated feature of the sawtooth lattice is the appearance of flat bands, setting $J_{A B} / J_{A A}=\sqrt{2}$ and $\phi=0$ [37]. Here we are, however, interested in the implications of a nontrivial phase, $\phi \neq 0$, leading to nonsymmetric bands [see Fig. 1(b)], due to the explicit breaking of time reversal $\left[H_{\mathrm{ST}} \neq H_{\mathrm{ST}}^{*}\right.$, which implies $h_{\mathrm{ST}}(k) \neq h_{\mathrm{ST}}(-k)$ ]. This cannot happen in a photonic system without loops since one can get rid of the phase $\phi$ by means of local transformations of the bosonic operators. Besides, there appears another gap between both bands unless $\phi= \pm \pi / 2$ [see again Fig. 1(b)] where the two bands touch at a single point $k= \pm \pi / 2$. These singular band gaps lead to exotic phenomenology in higher dimensions [38-40]. This is not the case here, since the coupling strength to the upper/lower band $\omega_{l / u}(k)$, defined by the functions $\theta_{k}, \varphi_{k}$, turn this point into a trivial band crossing, as we explain in the next section.

\section{QE RESONANT WITH $\omega_{u / l}(k)$}

We study the spontaneous decay of one qubit when $\Delta$ lies within the bands. The state at time $t$ reads 


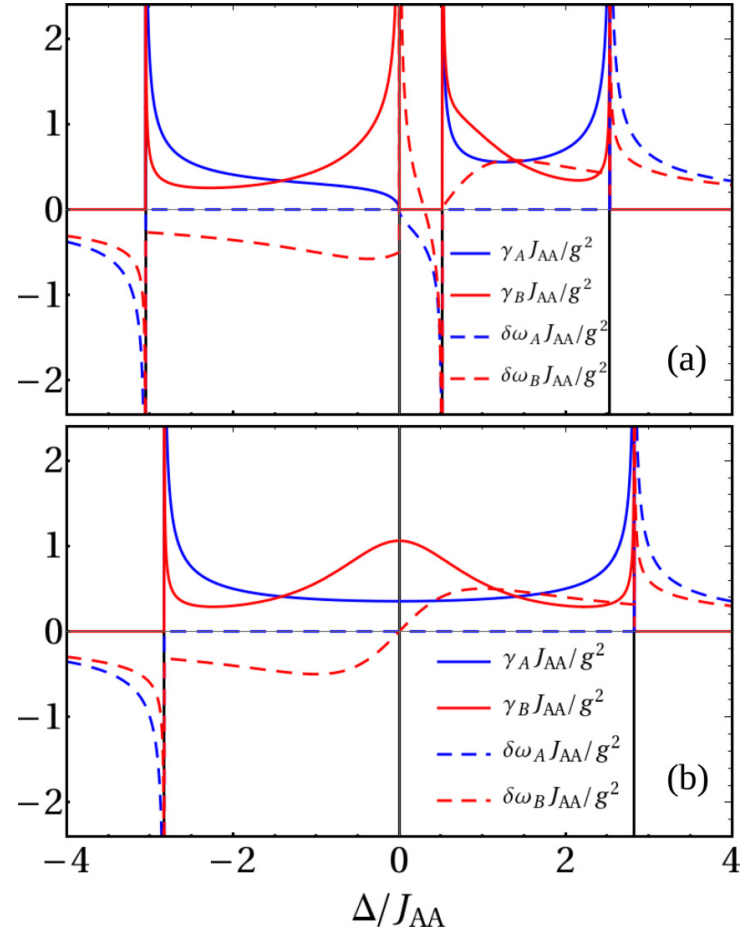

FIG. 2. Decay rate $\gamma_{D}=-2 \operatorname{Im} \Sigma_{e}^{D}$ (solid lines) and energy shift $\delta \omega_{D}(\Delta)=\operatorname{Re} \Sigma_{e}^{D}\left(\Delta+i 0^{+}\right)$(dashed lines) as a function of $\Delta$ for a qubit coupled to sublattice $A$ (blue) and $B$ (red), with $J_{A B}=J_{A A}$ and $\phi=\pi / 3$ (a) and $\phi=\pi / 2$ (b). The vertical black lines stand for the band limits.

$\left|\Psi_{D}(t)\right\rangle=e^{-i H t} \sigma^{+}|\mathrm{vac}\rangle$, where $|\mathrm{vac}\rangle$ is the vacuum state and $D$ stands for the sublattice the QE is coupled to. As the number of excitations $\mathscr{N} \equiv \sum_{n=1}^{N}\left(a_{n}^{\dagger} a_{n}+b_{n}^{\dagger} b_{n}\right)+\sigma^{+} \sigma^{-}$is a conserved quantity under the rotating-wave approximation (3), the state $\left|\Psi_{D}(t)\right\rangle$ can be spanned in the single-excitation subspace:

$$
\left|\Psi_{D}(t)\right\rangle=\left\{c_{e}^{D}(t) \sigma^{+}+\sum_{k}\left[c_{u}^{D}(k, t) \hat{u}_{k}^{\dagger}+c_{l}^{D}(k, t) \hat{l}_{k}^{\dagger}\right]\right\}|0\rangle .
$$

We can then calculate the dynamics numerically or semianalytically using the resolvent method [36], where $c_{e}^{D}(t)$ is obtained as $c_{e}^{D}(t)=\mathscr{L}^{-1}\left[G_{e}(z)\right]$, being $\mathscr{L}$ the Laplace transform and $G_{e}(z)$ the QE Green Function. The latter reads $G_{e}(z)=1 /\left[z+\Sigma_{e}^{D}(z)\right]$, being the self-energy $\Sigma_{e}^{D}(z)$ (see Appendix B)

$$
\Sigma_{e}^{D}(z)=\sum_{k} \sum_{\alpha=u, l} \frac{\left|\left\langle 0\left|\alpha_{k} H_{\text {int }} \sigma^{+}\right| 0\right\rangle\right|^{2}}{z-\omega_{\alpha}(k)} .
$$

Within the Markovian approximation $z$ can be replaced by $z=\Delta+i 0^{+}$when doing the inverse Laplace, yielding $c_{e}^{D}(t) \simeq e^{-i \Sigma_{e}^{D}\left(\Delta+i 0^{+}\right) t}$, where $\Sigma_{e}^{D}\left(\Delta+i 0^{+}\right)=\delta \omega_{D}-i \gamma_{D} / 2$. Therefore, $\delta \omega_{D}$ and $\gamma_{D}$ correspond to the renormalization of the excited state frequency and line width, respectively. In Fig. 2 we plot these quantities for $\phi=\pi / 3$ and $\phi=\pi / 2$ [Figs. 2(a) and 2(b), respectively]. Both $\delta \omega_{D}$ and $\gamma_{D}$ depends on the sublattice the $\mathrm{QE}$ is coupled. Besides, as usual with this kind of system, $\gamma_{D}$ diverges in the band limits; there is

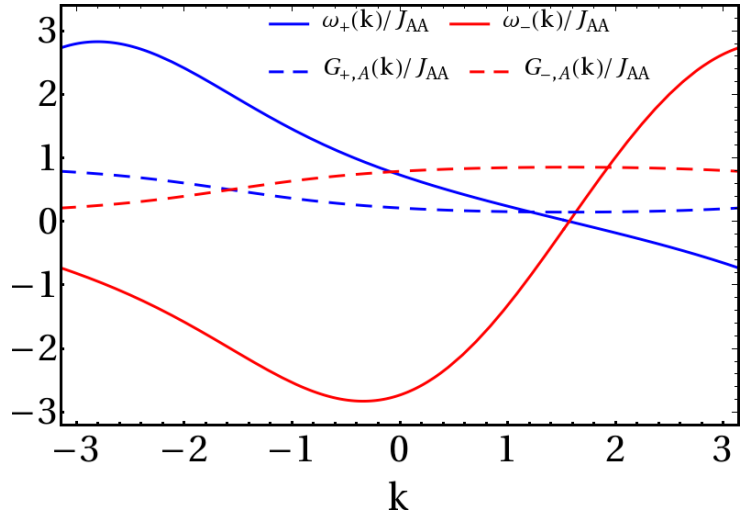

FIG. 3. Coupling $G_{ \pm, A}(k)$ between a qubit placed at sublattice $A$ and $\omega_{ \pm}(k)$ for $\phi=\pi / 2$ and $J_{A A}=J_{A B}$. As seen, the couplings $G_{ \pm, A}(k)$ are not discontinuous at $k=\pi / 2$.

an exception for $\phi \neq \pi / 2$, where $\gamma_{A}$ actually tends to 0 when $\Delta$ matches the the upper (lower) band edge of $\omega_{l}(k)\left[\omega_{u}(k)\right]$ for $\phi \in[0, \pi / 2)[(\pi / 2, \pi]]$. Similar behavior was also found in two-dimensional photonic crystals without sublattice symmetry [41].

When $\phi=\pi / 2$, the self-energy (both the real and the imaginary parts) is a smooth function for energies in the band. This predicts that the spontaneous emission by a $\mathrm{QE}$ will undergo an exponential decay. Consequently, the singular band gap here does not imply exotic dynamics, contrarily to the case of other photonic reservoirs in higher dimensions [38-40]. This is better understood studying how the QE couples to the bands for $\phi=\pi / 2$. We define two new bands $\omega_{ \pm}(k)$, together with the corresponding qubit-band couplings $G_{ \pm, D}(k)$ (see Appendix C) as

$$
\begin{aligned}
\omega_{ \pm}(k) & \equiv \begin{cases}\omega_{u}(k) & \text { if } k<\pi / 2 \\
\omega_{l}(k) & \text { if } k>\pi / 2\end{cases} \\
G_{ \pm, D}(k) & \equiv\left\{\begin{array}{ll}
G_{u, D}(k) & \text { if } k<\pi / 2 \\
G_{l, D}(k) & \text { if } k>\pi / 2
\end{array},\right.
\end{aligned}
$$

being $G_{u / l, D}(k)$ the coupling to the upper/lower band when the $\mathrm{QE}$ is coupled to the sublattice $D$ (see again Appendix C). Even if the results do not depend on the sublattice the QE couples to, we consider for the sake of simplicity that the QE is coupled to $A$. We plot both $\omega_{ \pm}(k)$ and $G_{ \pm, A}(k)$ in Fig. 3 for $\phi=\pi / 2$. As seen, these bands $\omega_{ \pm}(k)$ do not have any kink: They are smooth functions and just cross at $k=\pi / 2$. The couplings are also smooth. In conclusion, the apparent nonanalytical behavior is actually an artifact of the definition of the bands.

When calculating the exact QE dynamics (not shown), we find band-edge-related phenomena such as fractional and power-law decays in the long-time limit, similarly to those appearing in other photonic materials [38,41-52]. We focus instead on the emitted photons, which displays very distinctive features from other photonic baths. We plot in Fig. 4(a) a snapshot of the photon population in real space for a situation where the emission is highly directional, which corresponds to a $\mathrm{QE}$ coupled to the $B$ sublattice with parameters $J_{A B}=$ $0.2 J_{A A}, \phi=1.5$, and $\Delta=-0.5 J_{A A}$. We emphasize that, even though the bath breaks the $\pm k$ symmetry for any $\phi \neq 0$, the 

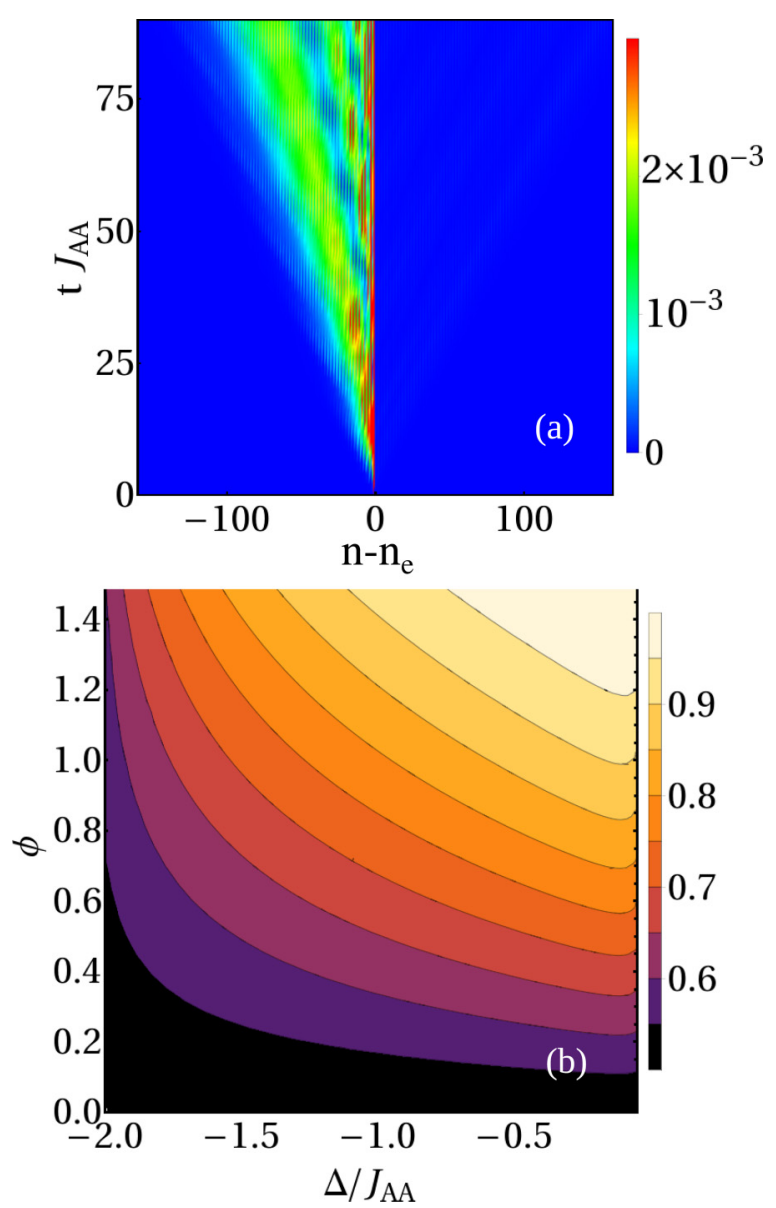

FIG. 4. (a) Emitted photon in positions as a function of time, $\left\langle a_{n}^{\dagger} a_{n}\right\rangle(t)$ and $\left\langle b_{n}^{\dagger} b_{n}\right\rangle(t)$, when the qubit is coupled to $B$ for $J_{A B}=$ $0.2 J_{A A}, \phi=1.5, \Delta=-0.5 J_{A A}, g=0.1 J_{A A}$, and bath size $N=200$. The qubit is at $n=n_{e}$. (b) Directionality ratio $R_{L}^{B}$ for $J_{A B}=0.2 J_{A A}$ as a function of $\Delta$ and $\phi$.

degree of directionality depends strongly on the parameters, especially $\Delta$. That is, $\phi \neq 0$ is a necessary but not sufficient condition for chiral emission.

Let us further understand the origin and possibilities of the directional emission in this system by considering that the QE is resonant with $\omega_{l}(k)$ and taking the limit when $g$ is small enough such that we are in the Markov regime. In this regime, the dynamics is dominated by the resonant $k$-modes defined by $\omega_{l}\left(k_{R / L}\right)=\Delta$, where $k_{R / L}$ correspond to right- and leftmoving photons, respectively [see Fig. 1(b)]. Furthermore, within each direction the excitations split between photons propagating in the $A$ or $B$ sublattices. Thus, the decay rate $\gamma_{D}$ introduced as the imaginary part of the self-energy [Eq. (9)] can be separated into four contributions:

$$
\gamma_{D}=\Gamma_{a}^{D}\left(k_{R}\right)+\Gamma_{a}^{D}\left(k_{L}\right)+\Gamma_{b}^{D}\left(k_{R}\right)+\Gamma_{b}^{D}\left(k_{L}\right),
$$

where $\Gamma_{\alpha}^{D}(k)$ denotes the decay rate into the $\alpha$ sublattice at momentum $k$ for a QE coupled to the $D$ sublattice, reading

$$
\begin{gathered}
\Gamma_{a(b)}^{A(B)}(k)=\frac{\left|\sin \left(\theta_{k}\right)\right|^{4}\left(\left|\cos \left(\theta_{k}\right)\right|^{4}\right)}{\left|v_{l}(k)\right|}, \\
\Gamma_{b}^{A}(k)=\frac{\left|\sin \left(\theta_{k}\right)\right|^{2}\left|\cos \left(\theta_{k}\right)\right|^{2}}{\left|v_{l}(k)\right|}=\Gamma_{a}^{B}(k)
\end{gathered}
$$

where $v_{l}(k)$ is the group velocity in the lower band, $v_{l}(k)=$ $\partial_{k} \omega_{l}(k)$ and $\theta_{k}$ was defined in (6) (see also Appendix A). We define a global directionality ratio:

$$
R_{L / R}^{D}=\frac{\sum_{\alpha} \Gamma_{\alpha}^{D}\left(k_{R / L}\right)}{\sum_{\alpha}\left[\Gamma_{\alpha}^{D}\left(k_{R}\right)+\Gamma_{\alpha}^{D}\left(k_{L}\right)\right]}
$$

with $\alpha=a, b$, that tell us the ratio of light emitted in the left/right side in both sublattices, and a local one which distinguishes between sublattices $R_{R / L, a / b}^{D}$ with the same expressions but without summing in $\alpha$.

In Fig. 4(b) we plot $R_{L}^{B}$ as a function of $\Delta$ and $\phi>0$ for a tunneling $J_{A B}=0.2 J_{A A}$. We observe that we can find nonreciprocal emission, that is, $R_{\alpha}^{D}>1 / 2$ for any $\phi \neq 0$. However, in order to find $R_{L}^{B} \approx 1$ one has to take the limit $J_{A B} / J_{A A} \ll 1$, $\phi \lesssim \pi / 2$, and $\Delta \lesssim 0$. In this regime, the slope of $\omega_{l}(k)$ around $k=\pi / 2$ is drastically different for the resonant momenta $k_{R}$ and $k_{L}$, yielding a density of states which is much larger for $k_{L}$ than for $k_{R}$. Besides, $\theta_{k}$ is such that the numerators of $\Gamma_{\alpha}^{B}(k)$ are much larger for $k_{L}$ than for $k_{R}$, which actually reinforces the previous argument. Notice that the directional emission can be kept for more than $95 \%$ for variations of $\phi$ and $\Delta$ around $10 \%$ of the optimal value.

If the $\mathrm{QE}$ couples to the $A$ sublattice instead, the global emission is not directional: $R_{L / R}^{A}=1 / 2$. However, locally in each sublattice, the emission can be made very directional, $R_{L, b}^{A}, R_{R, a}^{A} \approx 1$. We illustrate this fact in Fig. 5, where we see that once again we get chirality for $\phi \rightarrow \pi / 2$ and $\Delta \rightarrow 0$. This is possible because in that case the coefficients $\theta_{k_{R / L}}$ are such that the directionality is reversed for emission into the $a$ modes: $\Gamma_{a}^{A}\left(k_{R}\right) \gg \Gamma_{a}^{A}\left(k_{L}\right)$, compensating the effect of the density of states. We show a snapshot of wave packets emitted into opposite directions in Fig. 6: As seen, the qubit emits mainly into right-/left-propagating $a / b$ modes. To our knowledge, this is the first time this sublattice-dependent chirality has been reported in the literature.

\section{QES OUTSIDE OF THE BAND: TUNABLE COMPLEX INTERACTIONS}

We focus now on the regime where $\Delta \notin \omega_{l / u}(k)$, such that the physics is dominated by the bound states (BSs) [52-66]. In the single-excitation subspace, the BS wave function of one emitter coupled to the $D$ sublattice reads

$$
\left|\Psi_{m}^{D}\right\rangle=\sum_{n}\left[c_{m, a}^{D}(n) a_{n}^{\dagger}+c_{m, b}^{D}(n) b_{n}^{\dagger}\right]|0\rangle+c_{m, e}^{D} \sigma^{+}|0\rangle,
$$

where $m=-1,0,1$ denotes the different BSs that can appear in the upper/middle/lower band gap, respectively. Their wave functions and energies are obtained from $H\left|\Psi_{m}^{D}\right\rangle=E_{m}^{D}\left|\Psi_{m}^{D}\right\rangle$ with $E_{m}^{D} \notin \omega_{l, u}(k)$ (see Appendix D). There always exists a BS $\left|\Psi_{\mp 1}^{D}\right\rangle$ below [above] $\omega_{l[u]}(k)$, because the self-energy always diverges at these band edges [62,64], such that the interaction with the bath is able to push one state out of the band; however, in the middle band gap, an extra BS $\left|\Psi_{0}^{D}\right\rangle$ emerges when $\Delta>0,|\phi|<\pi / 2$ or $\Delta<0$ and $\phi \in(-\pi,-\pi / 2) \cup$ $(\pi / 2, \pi)$ for $D=B$ or $A$, respectively (see Appendix D). The reason of this condition is the finite value of $\Sigma_{e}^{D}(z)$ in one of the band edges, as shown in Fig. 2(a), which defines a critical detuning for the existence of the BS. 

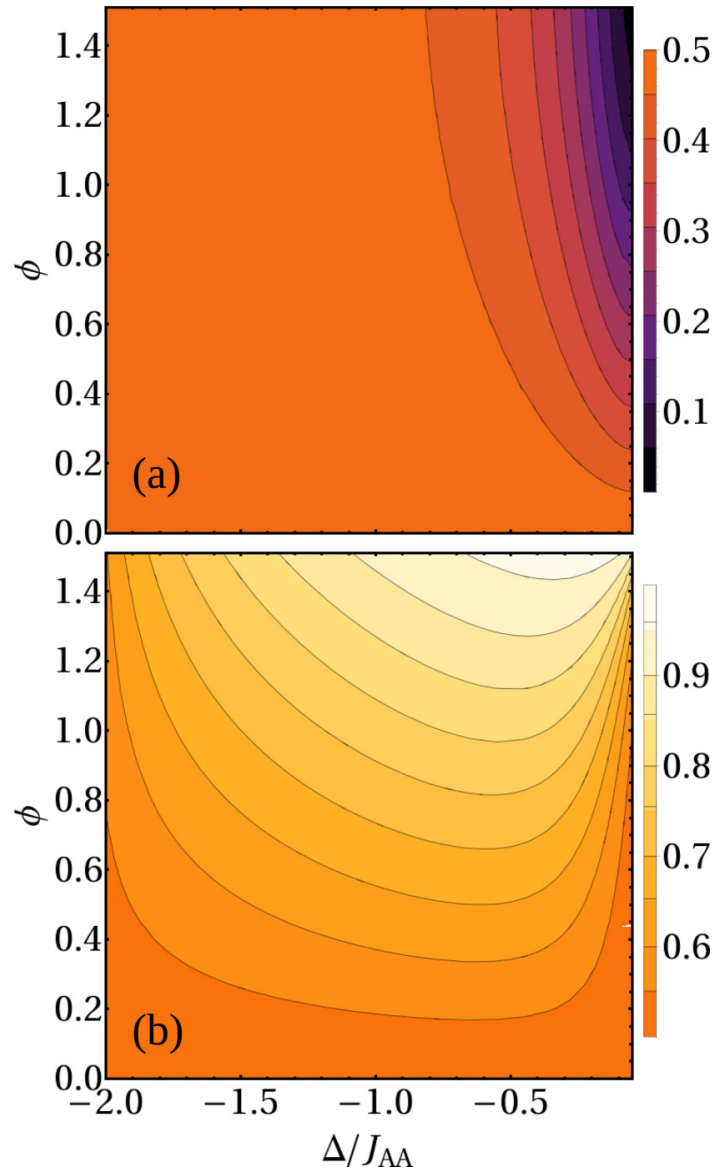

FIG. 5. Directionality ratio $R_{L, a / b}^{A}$ in panels (a) and (b), respectively, for $J_{A B}=0.2 J_{A A}$. Notice that the scale of both panels is different: $(0,0.5)$ in (a) and $(0.5,1)$ in (b).

To illustrate the main features of these BSs, we plot in Fig. 7 their wave function coefficients in momenta and positions for the interband BS $\left|\Psi_{0}^{D}\right\rangle$. (1) Contrarily to what happens in emission, the absolute value of the wave function $\left|c_{m, \alpha}^{D}(n)\right|$ is always symmetrically distributed around the $\mathrm{QE}$ no matter the band gap or parameters considered; (2) as it occurs with other photonic lattices [52-66], the BS are exponentially localized around the emitter with a localization

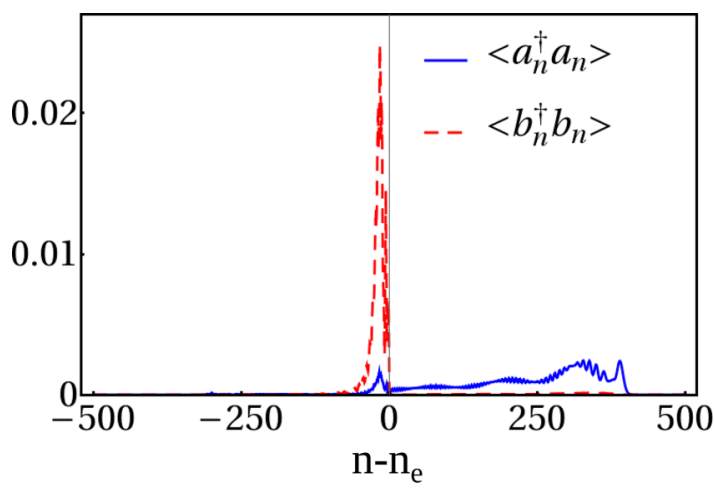

FIG. 6. Emitted wave packet when the qubit is coupled to $A$ for $J_{A B}=0.2 J_{A A}, \phi=1.5, \Delta=-0.1 J_{A A}, g=0.1 J_{A A}, N=1000$, and $t J_{A A}=200$.

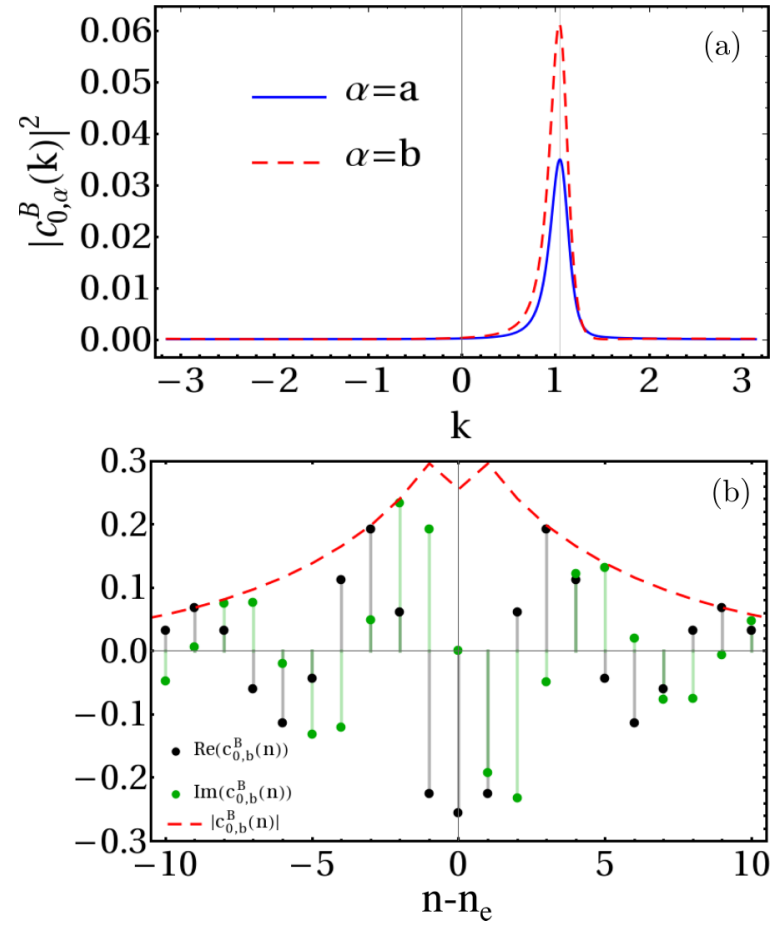

FIG. 7. Interband BS $\left|\Psi_{0}^{B}\right\rangle$ for a qubit coupled to $B$ for $J_{A A}=$ $J_{A B}=1, \phi=2.094, \Delta=-0.01$, and $g=0.1$. In panel (a) we plot the square modulus of the wave functions in momenta in both sublattices, $\left|c_{0, a / b}^{B}(k)\right|^{2}$, whereas we show the absolute value (red dashed line) and the real (black dots) and imaginary (green dots) parts of the coefficients in positions for the $b$ modes in panel (b). The wave functions in $k$ space are centered at $k=\pi / 3$, so the periodicity in positions is 6 . Notice that the qubit is placed at $n=n_{e}$.

length which can be tuned: the closer $E_{m}^{D}$ lies to one of the band edges, the less localized the BS is; and (3) the novelty is that the BSs acquire a tunable complex phase $c_{m, \alpha}^{D}(n) \propto e^{i \varphi_{m}^{D} n}$. For small $g, \varphi_{m}^{D}$ matches the position of the band edge closest to $\Delta$, which turns out to depend on $J_{A B} / J_{A A}$ and $\phi$, as seen in Fig. 1(b), e.g., the minimum of the upper band runs from 0 to $\pi / 2$, so the phase of the interband BS can be tuned in this range provided $\Delta$ tends to this band edge. In the example of Fig. 7, the momentum of the closest band edge occurs at $k_{\text {edge }} \simeq \pi / 3$, so $\left|c_{0, \alpha}^{B}(k)\right|^{2}$ is distributed around $\pi / 3$ [Fig. 7(a)] and $c_{0, \alpha}^{B}(n)$ has periodicity $2 \pi /(\pi / 3)=6$ [Fig. 7(b)].

When many emitters couple to the bath, the BS mediate interactions between QEs, which can be harnessed to simulate spin models with tunable interactions, allowing for the study of many-body physics, phase transitions, etc. (e.g., see Refs. [38,38,40,62,64,66-68]).

In the Markovian approximation, that is, when the coupling strength between the qubits and the bath is small compared to the gap between $\Delta$ and the bands (recall that we are considering the regime where $\Delta$ is not embedded in the photonic modes) and also compared to the width of both bands, an effective Hamiltonian for the qubits can be derived $[67,68]$ :

$$
H_{\mathrm{qb}}=\sum_{i<j}\left[J_{i j}^{D_{i j} D_{j}} \sigma_{i}^{+} \sigma_{j}^{-}+\text {H.c. }\right] \text {, }
$$




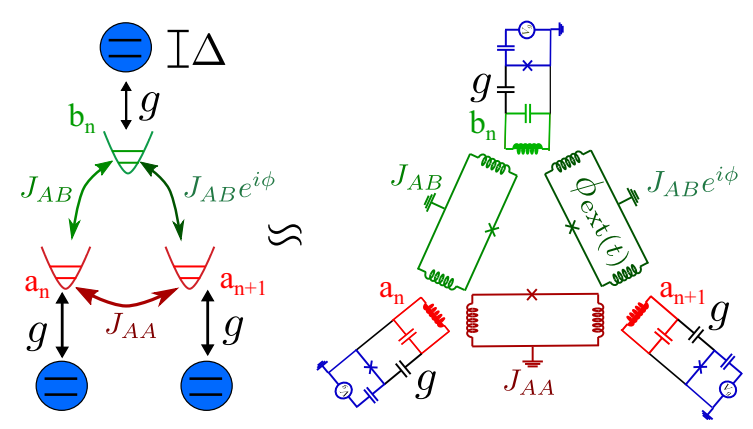

FIG. 8. On the left-hand side, we show a plaquette of the sawtooth lattice coupled to three qubits. On the right-hand side, the proposal for the implementation. The qubits are the blue parts. In black, we show the qubit-resonator couplings. Both the $a / b$ resonators and the resonator-resonator couplings are in red and green.

being $D_{i}$ the sublattice the $i$ th qubit is coupled to. Here $J_{i j}^{D_{i} D_{j}}$ is proportional to the two-qubit self-energy (see Appendix E) and it inherits the shape of the BS wave function with energy $\Delta$, i.e., $J_{i j}^{A A / B B} \propto c_{a / b}^{A / B}\left(r_{i j}\right)$ and $J_{i j}^{A B} \propto c_{b}^{A}\left(r_{i j}\right)$. This has several implications. First, qubit-qubit effective interactions decay exponentially with the distance between qubits, being the range of the interactions tunable; in particular, it is a decreasing function of the gap between $\Delta$ and the closest band and it diverges when this gap closes. Besides, we can also tune its phase, which, as in the sawtooth bath, is physically relevant (see Appendix D), because the geometry of the effective spin lattice has closed loops. This allows us to tune both the periodicity of the interactions [see, for instance, Fig. 7(b), where periodicity is 6], which may give rise to qualitatively different spin phases. Even richer many-body dynamics will appear in the nonperturbative regime replacing spins by polaritons [65].

\section{IMPLEMENTATION}

As the physics of Eq. (2) does not rely on polarization, it can be obtained in platforms beyond optical ones [7,8,1012] such as cold atoms in state-dependent lattices [69-71], where such complex loops have already been engineered [72], or circuit QED platforms [73-79]. In Fig. 8 we show a proposal to design this geometry with complex couplings using superconducting qubits as implemented for a single loop in Ref. [80]. For the qubit-resonator interaction, we assume that the qubit is capacitively coupled to an LC resonator, whereas the resonators are inductively coupled via a SQUIDtype loop based on Josephson junctions $[81,82]$. The junctions are controlled via an external flux $\phi_{\text {ext }}(t)$ leading to the timedependent coupling Hamiltonian between two resonators:

$$
H_{\mathrm{aux}}=\sum_{i=1,2} \omega_{i} a_{i}^{\dagger} a_{i}+J(t)\left(a_{1}^{\dagger}+a_{1}\right)\left(a_{2}^{\dagger}+a_{2}\right) .
$$

Setting $\omega_{1}=\omega, \omega_{2}=\omega+\delta$, and $J(t)=J \cos (\delta t+\phi)$, and assuming $J, \delta \ll \omega$, we can average out the time dependence of $J(t)$, getting the Hamiltonian we want to simulate; cf. Eq. (2) [83]. For the implemented loop of Ref. [80], they measure couplings of the order of $4.1 \mathrm{MHz}$ (i.e.. $J / \omega \sim 10^{-3}$ ). Thus, concatenating several of these cells and placing selectively the qubits, as done in Refs. [84,85] for simple coupledcavity arrays, one can explore the phenomena predicted in this paper.

Thanks to the tunability of cQED implementations, one can explore different regimes of parameters (e.g., see Refs. [78,79]); in particular, it is possible to study the Markovian regime, on which the results presented here are based. It would be interesting also to study other regimes, e.g., the ultrastrong coupling, which has been found in waveguide QED [86], and whose consequences in chiral light-matter interactions have been recently explored [87].

\section{CONCLUSIONS}

We have studied the properties of QEs coupled to a minimal one-dimensional model breaking time-reversal symmetry: the photonic sawtooth lattice. When the emitters are resonant with the band they decay in an asymmetric fashion into left-/right-moving modes. We identified regimes of quasiperfect directionality, or more exotic ones in which the emitter decays in both directions but to a different sublattice. Thus, these systems can be an alternative way of exploring chiral quantum optics without polarization or moving emitters. Besides, when the emitter frequency lies in a band gap we have found the emergence of BSs whose not only their spatial range, but also their complex phase can be tuned through the system parameters. Since these BSs mediate interactions between emitters when many of them couple to the bath, our setup provides access to the simulation of a large class of spin models with complex interactions. Furthermore, we discussed an implementation to observe such phenomenology based on superconducting technologies.

\section{ACKNOWLEDGMENTS}

E.S.B. acknowledges ERC Advanced Grant QUENOCOBA under the EU Horizon 2020 program (Grant Agreement No. 742102). A.G.T. and D.Z. acknowledge support from CSIC Research Platform PTI-001. A.G.T. acknowledges funding from the national project PGC2018-094792-BI00 from Ministerio de Ciencia e Innovación.

\section{APPENDIX A: DIAGONALIZATION OF THE SAWTOOTH LATTICE}

In this Appendix, we give some details on the diagonalization of the sawtooth Hamiltonian [Eq. (2)]. The bosonic operators which diagonalize the model, $\hat{u}_{k} / \hat{l}_{k}$ [see Eq. (6)] are related to $\hat{a}_{k}$ and $\hat{b}_{k}$ by means of a unitary transformation $P_{k}$. The latter reads

$$
P_{k}=\left(\begin{array}{cc}
\cos \left(\theta_{k}\right) e^{i \varphi_{k}} & \sin \left(\theta_{k}\right) e^{i \varphi_{k}} \\
-\sin \left(\theta_{k}\right) & \cos \left(\theta_{k}\right)
\end{array}\right)=\left(\begin{array}{cc}
N_{u}(k) f^{*}(k, \phi) & N_{l}(k) f^{*}(k, \phi) \\
N_{u}(k)\left(\omega_{u}(k)+2 J_{A A} \cos k\right) & N_{l}(k)\left(\omega_{l}(k)+2 J_{A A} \cos k\right)
\end{array}\right)
$$


where $N_{u / l}(k)$ is a normalization factor

$$
N_{u / l}(k)=\frac{1}{\sqrt{|f(k, \phi)|^{2}+\left[\omega_{u / l}(k)+2 J_{A A} \cos k\right]^{2}}} .
$$

\section{APPENDIX B: SINGLE-QUBIT SELF-ENERGY}

To compute $c_{e}^{D}(t)$, we use the resolvent operator method [36], which tells us that the probability amplitude can be computed as

$$
c_{e}^{D}(t)=-\frac{1}{2 \pi i} \int_{-\infty}^{\infty} d E G_{e}^{D}\left(E+i 0^{+}\right) e^{-i E t},
$$

where $G_{e}^{D}(z)$ is the single-qubit Green function when it is coupled to the sublattice $D$ :

$$
G_{e}^{D}(z)=\frac{1}{z-\Delta-\Sigma_{e}^{D}(z)},
$$

where $\Sigma_{e}^{D}(z)$ the so-called self-energy. In this section, we derive the expressions for the single-qubit self-energy when the qubit is locally coupled to $A$ or $B$. The $\Sigma_{e}^{D}(z)$ of our two-band model reads

$$
\Sigma_{e}^{D}(z)=\sum_{k} \sum_{\alpha=u, l} \frac{\left|\left\langle 0\left|\alpha_{k} H_{\mathrm{int}} \sigma^{+}\right| 0\right\rangle\right|^{2}}{z-\omega_{\alpha}(k)} .
$$

Considering $H_{\text {int }}$ [Eq. (3)] for a single qubit) coupled to $A$ or $B$ and taking into account the relation between $\left(u_{k}, l_{k}\right)$ and $\left(a_{k}, b_{k}\right)$ [see Eqs. (6) and (A1)] and the expressions for $\omega_{u / l}(k)$ [see Eq. (7)]:

$$
\begin{aligned}
& \Sigma_{e}^{A}(z)=\frac{g^{2}}{2 \pi} \int_{-\pi}^{\pi} d k \frac{z}{z^{2}+2 z J_{A A} \cos k-|f(k, \phi)|^{2}}, \\
& \Sigma_{e}^{B}(z)=\frac{g^{2}}{2 \pi} \int_{-\pi}^{\pi} d k \frac{z+2 J_{A A} \cos k}{z^{2}+2 z J_{A A} \cos k-|f(k, \phi)|^{2}} .
\end{aligned}
$$

We take here the thermodynamic limit: $N \rightarrow \infty$. One can solve these integrals by means of the change of variable $y \equiv e^{i k}$. The integration domain is now the unit circle in the complex plane:

$$
\begin{gathered}
\Sigma_{e}^{A}(z)=\frac{g^{2}}{2 \pi i} \oint d y \frac{z J_{A A}}{\left(z J_{A A}-J_{A B}^{2} e^{i \phi}\right)\left(y-y_{+}\right)\left(y-y_{-}\right)}, \\
\Sigma_{e}^{B}(z)=\frac{g^{2}}{2 \pi i} \oint d y \frac{J_{A A} y^{2}+z y+J_{A A}}{\left(z J_{A A}-J_{A B}^{2} e^{i \phi}\right) y\left(y-y_{+}\right)\left(y-y_{-}\right)},
\end{gathered}
$$

where $y_{ \pm}$are

$$
y_{ \pm}=\frac{2 J_{A B}^{2}-z^{2} \pm \sqrt{\left(2 J_{A B}^{2}-z^{2}\right)^{2}-4\left(z^{2} J_{A A}^{2}+J_{A B}^{4}-2 z J_{A A} J_{A B}^{2} \cos \phi\right)}}{2\left(z J_{A A}-J_{A B}^{2} e^{i \phi}\right)} .
$$

We define $y_{\min / \max }$ as the minimum/maximum of $\left\{y_{-}, y_{+}\right\}$ with respect to the absolute values $\left|y_{ \pm}\right|$. Applying the Cauchy's residue theorem and taking into account that $\left(\left|y_{+}\right|-\right.$ $1)\left(\left|y_{-}\right|-1\right)<1$ for all $z \in \mathbb{C}$ with $\operatorname{Im}(z) \neq 0$ :

$$
\begin{gathered}
\Sigma_{e}^{A}(z)=\frac{g^{2} z \operatorname{sign}\left(\left|y_{-}\right|-\left|y_{+}\right|\right)}{\left(z J_{A A}-J_{A B}^{2} e^{i \phi}\right)\left(y_{+}-y_{-}\right)}, \\
\Sigma_{e}^{B}(z)=\frac{g^{2} J_{A A}}{z J_{A A}-J_{A B}^{2} e^{i \phi}}\left[\frac{1}{y_{+} y_{-}}+\frac{y_{\min }^{2}+\left(z / J_{A A}\right) y_{\min }+1}{y_{\min }\left(y_{\min }-y_{\max }\right)}\right] .
\end{gathered}
$$

For instance, if we consider that $\Delta$ is embedded in the lower band, it is straightforward to derive Eqs. (13) and (14) from Eqs. (B9) and (B10).

\section{APPENDIX C: QUBIT-BAND COUPLINGS}

Here we write the qubit-band coupling for both bands.

Let us consider the interaction Hamiltonian $H_{\text {int }}$ [Eq. (3)] for a single qubit. For the sake of simplicity, the qubit will be coupled to $A$; it is because of this that we will denote the interaction Hamiltonian as $H_{\text {int }}^{A}$ in this Appendix. We write this Hamiltonian in terms of $u_{k}$ and $l_{k}$ [see Eq. (6)]:

$H_{\text {int }}^{A}=\frac{g}{\sqrt{N}} \sigma^{+} \sum_{k} e^{i k x_{0}}\left[\cos \left(\theta_{k}\right) e^{i \varphi_{k}} \hat{u}_{k}+\sin \left(\theta_{k}\right) e^{i \phi_{k}} \hat{l}_{k}\right]+$ H.c.,

where $x_{0}$ is the position of the qubit and $\cos / \sin \left(\theta_{k}\right)$ are the matrix elements of the unitary transformation $P_{k}$ [see
Eq. (A1)]. The latter determines the coupling strength to each band: $G_{u, A}(k)=\left|\cos \left(\theta_{k}\right)\right|^{2}$ and $G_{l, A}(k)=\left|\sin \left(\theta_{k}\right)\right|^{2}$, up to the density of states, which is given by $1 /\left|\partial \omega_{u / l}(k)\right|$.

\section{APPENDIX D: BOUND STATES}

In this section, we discuss the existence conditions of the bound states (BSs) and we compute their wave functions in real space.

As mentioned in the main text, we have to impose the eigenvalue equation $H\left|\Psi_{m}^{D}\right\rangle=E_{m}^{D}\left|\Psi_{m}^{D}\right\rangle$ with the energy $E_{m}^{D}$ outside of the bands. This can be mapped into finding the roots of the following function:

$$
F_{D}(E) \equiv E-\Delta-\Sigma_{e}^{D}(E),
$$

with $E \notin \omega_{l, u}(k)$ [64]. It can be easily proved from Eq. (9) that $\Sigma_{e}^{D}(E)$ is a decreasing function, so $F_{D}(E)$ is an increasing function. Besides, $\lim _{E \rightarrow \pm \infty} F_{D}(E)= \pm \infty$. Then, according to the behavior of $\Sigma_{e}^{D}(E)$ in the band edges, we can figure out whether there exists or not a bound state in each of the band gaps:

(1) A BS exists with $E_{\mathrm{bs}}<\omega_{l}(k)\left[E_{\mathrm{bs}}>\omega_{l}(k)\right]$ for all the values of the parameters if and only if $F_{D}(E)>(<) 0$ when $E$ tends to the minimum of $\omega_{l}(k)$ [maximum of $\omega_{u}(k)$ ]. We plot $\Sigma_{e}^{D}(E)$ for $E$ outside of the band in Fig. 2 and show that it diverges in the the lowest/highest energy band edge, which guarantees the aforementioned conditions, so the existence of two BS below $\omega_{l}(k)$ and over $\omega_{u}(k)$, which we label as $\left|\Psi_{-1}^{D}\right\rangle$ and $\left|\Psi_{+1}^{D}\right\rangle$, respectively. 
(2) The situation is different in the middle band gap. In Fig. 2(a) we observe when the QE is coupled to the B lattice, the self-energy diverges in both the upper/lower middle band edges. Thus, a middle BS, $\left|\Psi_{0}^{B}\right\rangle$, always exists. On the other hand, if the emitter is coupled to $A$, the state $\left|\Psi_{0}^{A}\right\rangle$ exists if $\Delta>0$ when $|\phi|<\pi / 2$ because the self-energy $\Sigma_{e}^{A}(E)$ vanishes when $E$ tends to the maximum of $\omega_{l}(k)$. When $\phi \in(-\pi,-\pi / 2)$ or $\phi \in(\pi / 2, \pi)$, the existence condition is $\Delta<0$ (not shown).

Concerning the wave functions, if $D=A$, the coefficients read

$$
\begin{aligned}
& c_{m, a}^{A}(n)=\frac{g c_{e}^{A}}{2 \pi} \int_{-\pi}^{\pi} d k e^{i k n}\left[\frac{\left|\left(P_{k}\right)_{11}\right|^{2}}{E_{m}^{D}-\omega_{u}(k)}+\frac{\left|\left(P_{k}\right)_{12}\right|^{2}}{E_{m}^{A}-\omega_{l}(k)}\right], \\
& c_{m, b}^{A}(n)=\frac{g c_{e}^{A}}{2 \pi} \int_{-\pi}^{\pi} d k e^{i k n}\left[\frac{\left(P_{k}\right)_{21}\left(P_{k}\right)_{11}^{*}}{E_{m}^{A}-\omega_{u}(k)}+\frac{\left(P_{k}\right)_{22}\left(P_{k}\right)_{12}^{*}}{E_{m}^{A}-\omega_{l}(k)}\right],
\end{aligned}
$$

where $E_{m}^{A}$ is the energy of $\left|\Psi_{\mathrm{m}, \mathrm{bs}}^{A}\right\rangle$ and $c_{e}^{A}$ is obtained imposing the normalization condition. Doing the math,

$$
\begin{gathered}
c_{m, a}^{A}(n)=\frac{g c_{e}}{2 \pi} \int_{-\pi}^{\pi} d k \frac{e^{i k n} E_{\mathrm{m}}^{A}}{\left(E_{\mathrm{m}}^{A}\right)^{2}+J_{A A} 2 E_{\mathrm{m}}^{A} \cos k-|f(k, \phi)|^{2}}, \\
c_{m, b}^{A}(n)=\frac{-g c_{e}}{2 \pi} \int_{-\pi}^{\pi} d k \frac{e^{i k n} f^{*}(k, \phi)}{\left(E_{\mathrm{m}}^{A}\right)^{2}+2 J_{A A} E_{\mathrm{m}}^{A} \cos k-|f(k, \phi)|^{2}} .
\end{gathered}
$$

If the qubit is instead coupled to $B$,

$$
\begin{aligned}
& c_{m, a}^{B}(n)=\frac{g c_{e}^{B}}{2 \pi} \int_{-\pi}^{\pi} d k e^{i k n}\left[\frac{\left(P_{k}\right)_{11}\left(P_{k}\right)_{21}^{*}}{E_{m}^{D}-\omega_{u}(k)}+\frac{\left(P_{k}\right)_{12}\left(P_{k}\right)_{22}^{*}}{E_{m}^{B}-\omega_{l}(k)}\right], \\
& c_{m, b}^{B}(n)=\frac{g c_{e}^{B}}{2 \pi} \int_{-\pi}^{\pi} d k e^{i k n}\left[\frac{\left.\mid\left(P_{k}\right)_{21}\right)\left.\right|^{2}}{E_{m}^{B}-\omega_{u}(k)}+\frac{\left.\mid\left(P_{k}\right)_{22}\right)\left.\right|^{2}}{E_{m}^{B}-\omega_{l}(k)}\right],
\end{aligned}
$$

which becomes

$$
\begin{aligned}
& c_{m, a}^{B}(n)=\frac{-g c_{e}^{B}}{2 \pi} \int_{-\pi}^{\pi} d k \frac{e^{i k n} f(k, \phi)}{\left(E_{\mathrm{m}}^{B}\right)^{2}+2 J_{A A} E_{\mathrm{m}}^{B} \cos k-|f(k, \phi)|^{2}}, \\
& c_{m, b}^{B}(n)=\frac{g c_{e}^{B}}{2 \pi} \int_{-\pi}^{\pi} d k \frac{e^{i k n}\left(E_{\mathrm{m}}^{B}+2 \cos k\right)}{\left(E_{\mathrm{m}}^{B}\right)^{2}+2 J_{A A} E_{\mathrm{m}}^{B} \cos k-|f(k, \phi)|^{2}} .
\end{aligned}
$$

Notice that all these expressions look similar to $\Sigma_{e}^{D}(z)$ [see Eqs. (B4) and (B5)], so we can calculate the coefficients in terms of complex integrals [Eqs. (B6) and (B7)]. The change of variable is still $y=e^{i k}$ if $n \geqslant 1$, but $y=e^{-i k}$ if $n \leqslant-1$. In the first case, the poles of the integral are $y_{ \pm}$, while in the second are their complex conjugates $y_{ \pm}^{*}$.

\section{APPENDIX E: TWO-QUBIT SELF-ENERGY}

We derive here the expressions for the collective selfenergy $\Sigma_{c}^{D_{12}}$, which is proportional to the coefficients of the effective Hamiltonian (17). The computation is totally analogous to the single-qubit self-energy (see Sec. B). They read

$\Sigma_{c}^{A A}\left(z ; r_{12}\right)=\frac{g^{2}}{2 \pi} \int_{-\pi}^{\pi} d k \frac{e^{i k r_{12}} z}{z^{2}+2 z J_{A A} \cos k-|f(k, \phi)|^{2}}$,

$\Sigma_{c}^{B B}\left(z ; r_{12}\right)=\frac{g^{2}}{2 \pi} \int_{-\pi}^{\pi} d k \frac{e^{i k r_{12}}\left(z+2 J_{A A} \cos k\right)}{z^{2}+2 z J_{A A} \cos k-|f(k, \phi)|^{2}}$,

$\Sigma_{c}^{A B}\left(z ; r_{12}\right)=-\frac{g^{2}}{2 \pi} \int_{-\pi}^{\pi} d k \frac{e^{i k r_{12}} f^{*}(k, \phi)}{z^{2}+2 z J_{A A} \cos k-|f(k, \phi)|^{2}}$,

where $r_{12}=x_{2}-x_{1}$ is the relative position of the qubits. Notice that $\Sigma_{c}^{A A}\left(z ; r_{12}\right), \Sigma_{c}^{B B}\left(z ; r_{12}\right)$, and $\Sigma_{c}^{A B}\left(z ; r_{12}\right)$ are proportional to the bound-state coefficients $c_{m, a}^{A}\left(r_{12}\right)$, $c_{m, b}^{B}\left(r_{12}\right)$, and $c_{m, b}^{A}\left(r_{12}\right)$ respectively, by changing the bound-state energies $E_{m}^{D}$ by $z$ [see Eqs. (D4), (D9), and (D5)]. It is here where it becomes evident that the effective interactions are mediated by the bound states.

Finally, we can compute the accumulated phase of a closed loop in the effective spin lattice. For example, taking the parameters of Fig. $7\left(J_{A A}=J_{A B}=1, \phi=2.094, \Delta=\right.$ -0.01 , and $g=0.1$ ) and choosing the closed path $a \rightarrow a \rightarrow$ $b \rightarrow a$, this phase is $\arg \left[\Sigma_{c}^{A A}(\Delta ; 1)\right]+\arg \left[\Sigma_{c}^{A B}(\Delta ; 1)\right]+$ $\arg \left[\Sigma_{c}^{A B}(\Delta ;-1)\right] \simeq-1.22$. As it is nonzero, the effective models can simulate systems without time and parity invariance.
[1] D. Jalas, A. Petrov, M. Eich, W. Freude, S. Fan, Z. Yu, R. Baets, M. Popović, A. Melloni, J. D. Joannopoulos et al., Nat. Photonics 7, 579 (2013).

[2] C. Sayrin, C. Junge, R. Mitsch, B. Albrecht, D. O'Shea, P. Schneeweiss, J. Volz, and A. Rauschenbeutel, Phys. Rev. X 5, 041036 (2015).

[3] D. L. Sounas and A. Alù, Nat. Photonics 11, 774 (2017).

[4] C. Caloz, A. Alù, S. Tretyakov, D. Sounas, K. Achouri, and Z.-L. Deck-Léger, Phys. Rev. Appl. 10, 047001 (2018).
[5] K. Y. Bliokh, D. Smirnova, and F. Nori, Science 348, 1448 (2015).

[6] T. V. Mechelen and Z. Jacob, Optica 3, 118 (2016).

[7] R. Mitsch, C. Sayrin, B. Albrecht, P. Schneeweiss, and A. Rauschenbeutel, Nat. Commun. 5, 5713 (2014).

[8] J. Petersen, J. Volz, and A. Rauschenbeutel, Science 346, 67 (2014).

[9] K. Xia, G. Lu, G. Lin, Y. Cheng, Y. Niu, S. Gong, and J. Twamley, Phys. Rev. A 90, 043802 (2014). 
[10] M. Scheucher, A. Hilico, E. Will, J. Volz, and A. Rauschenbeutel, Science 354, 1577 (2016).

[11] I. Söllner, S. Mahmoodian, S. L. Hansen, L. Midolo, A. Javadi, G. Kiršanske, T. Pregnolato, H. El-Ella, E. H. Lee, J. D. Song et al., Nat. Nanotechnol. 10, 775 (2015).

[12] R. Coles, D. Price, J. Dixon, B. Royall, E. Clarke, P. Kok, M. Skolnick, A. Fox, and M. Makhonin, Nat. Commun. 7, 11183 (2016).

[13] P. Lodahl, S. Mahmoodian, S. Stobbe, A. Rauschenbeutel, P. Schneeweiss, J. Volz, H. Pichler, and P. Zoller, Nature (London) 541, 473 (2017).

[14] S. Mahmoodian, P. Lodahl, and A. S. Sørensen, Phys. Rev. Lett. 117, 240501 (2016).

[15] A. Metelmann and A. A. Clerk, Phys. Rev. X 5, 021025 (2015).

[16] D. Malz, L. D. Tóth, N. R. Bernier, A. K. Feofanov, T. J. Kippenberg, and A. Nunnenkamp, Phys. Rev. Lett. 120, 023601 (2018).

[17] T. Ramos, H. Pichler, A. J. Daley, and P. Zoller, Phys. Rev. Lett. 113, 237203 (2014).

[18] T. Ramos, B. Vermersch, P. Hauke, H. Pichler, and P. Zoller, Phys. Rev. A 93, 062104 (2016).

[19] B. Vermersch, T. Ramos, P. Hauke, and P. Zoller, Phys. Rev. A 93, 063830 (2016).

[20] H. Pichler, T. Ramos, A. J. Daley, and P. Zoller, Phys. Rev. A 91, 042116 (2015).

[21] P.-O. Guimond, H. Pichler, A. Rauschenbeutel, and P. Zoller, Phys. Rev. A 94, 033829 (2016).

[22] S. Mahmoodian, M. Čepulkovskis, S. Das, P. Lodahl, K. Hammerer, and A. S. Sørensen, Phys. Rev. Lett. 121, 143601 (2018).

[23] C. A. Downing, J. C. L. Carreño, F. P. Laussy, E. del Valle, and A. I. Fernández-Domínguez, Phys. Rev. Lett. 122, 057401 (2019).

[24] Z. Eldredge, P. Solano, D. Chang, and A. V. Gorshkov, Phys. Rev. A 94, 053855 (2016).

[25] K. M. Sliwa, M. Hatridge, A. Narla, S. Shankar, L. Frunzio, R. J. Schoelkopf, and M. H. Devoret, Phys. Rev. X 5, 041020 (2015).

[26] B. J. Chapman, E. I. Rosenthal, J. Kerckhoff, B. A. Moores, L. R. Vale, J. A. B. Mates, G. C. Hilton, K. Lalumière, A. Blais, and K. W. Lehnert, Phys. Rev. X 7, 041043 (2017).

[27] C. Müller, S. Guan, N. Vogt, J. H. Cole, and T. M. Stace, Phys. Rev. Lett. 120, 213602 (2018).

[28] L. Mercier de Lépinay, E. Damskägg, C. F. Ockeloen-Korppi, and M. A. Sillanpää, Phys. Rev. Appl. 11, 034027 (2019).

[29] A. Rosario Hamann, C. Müller, M. Jerger, M. Zanner, J. Combes, M. Pletyukhov, M. Weides, T. M. Stace, and A. Fedorov, Phys. Rev. Lett. 121, 123601 (2018).

[30] G. Calajó and P. Rabl, Phys. Rev. A 95, 043824 (2017).

[31] T. Nakamura and K. Kubo, Phys. Rev. B 53, 6393 (1996).

[32] M. Hyrkäs, V. Apaja, and M. Manninen, Phys. Rev. A 87, 023614 (2013).

[33] S. Flach, D. Leykam, J. D. Bodyfelt, P. Matthies, and A. S. Desyatnikov, Europhys. Lett. 105, 30001 (2014).

[34] If there are no loops, complex couplings have nonphysical implications.

[35] A. Seif, W. DeGottardi, K. Esfarjani, and M. Hafezi, Nat. Commun. 9, 1207 (2018).
[36] C. Cohen-Tannoudji, J. Dupont-Roc, and G. Grynberg, AtomPhoton Interactions: Basic Processes and Applications (WileyInterscience, New York, 1998).

[37] D. Leykam, A. Andreanov, and S. Flach, Adv. Phys. X 3 , 1473052 (2018).

[38] A. González-Tudela and J. I. Cirac, Phys. Rev. A 97, 043831 (2018).

[39] J. Perczel and M. D. Lukin, Phys. Rev. A 101, 033822 (2020).

[40] A. González-Tudela and J. I. Cirac, Quantum 2, 97 (2018).

[41] A. González-Tudela and F. Galve, ACS Photonics 6, 221 (2018).

[42] L. A. Khalfin, Soviet Phys. JETP 6, 1053 (1958).

[43] V. P. Bykov, Sov. J. Quantum Electron. 4, 861 (1975).

[44] L. Fonda, G. C. Ghirardi, and A. Rimini, Rep. Prog. Phys. 41, 587 (1978).

[45] M. N. Hack, Phys. Lett. A 90, 220 (1982).

[46] D. S. Onley and A. Kumar, Am. J. Phys. 60, 432 (1992).

[47] S. John and T. Quang, Phys. Rev. A 50, 1764 (1994).

[48] B. Gaveau and L. S. Schulmann, J. Phys. A 28, 7359 (1995).

[49] S. Garmon, T. Petrosky, L. Simine, and D. Segal, Fortschr. Phys. 61, 261 (2013).

[50] E. S. Redchenko and V. I. Yudson, Phys. Rev. A 90, 063829 (2014).

[51] F. Lombardo, F. Ciccarello, and G. M. Palma, Phys. Rev. A 89, 053826 (2014).

[52] E. Sánchez-Burillo, D. Zueco, L. Martín-Moreno, and J. J. García-Ripoll, Phys. Rev. A 96, 023831 (2017).

[53] S. John, Phys. Rev. Lett. 53, 2169 (1984).

[54] S. John, Phys. Rev. Lett. 58, 2486 (1987).

[55] Q.-J. Tong, J.-H. An, H.-G. Luo, and C. H. Oh, J. Phys. B 43, 155501 (2010).

[56] Q.-J. Tong, J.-H. An, H.-G. Luo, and C. H. Oh, Phys. Rev. A 81, 052330 (2010).

[57] P. Longo, P. Schmitteckert, and K. Busch, Phys. Rev. Lett. 104, 023602 (2010).

[58] P. Longo, P. Schmitteckert, and K. Busch, Phys. Rev. A 83, 063828 (2011).

[59] W. L. Yang, J.-H. An, C. Zhang, M. Feng, and C. H. Oh, Phys. Rev. A 87, 022312 (2013).

[60] Y.-Q. Lü, J.-H. An, X.-M. Chen, H.-G. Luo, and C. H. Oh, Phys. Rev. A 88, 012129 (2013).

[61] E. Sánchez-Burillo, D. Zueco, J. J. García-Ripoll, and L. Martín-Moreno, Phys. Rev. Lett. 113, 263604 (2014).

[62] G. Calajó, F. Ciccarello, D. Chang, and P. Rabl, Phys. Rev. A 93, 033833 (2016).

[63] G. Calajó, F. Ciccarello, D. Chang, and P. Rabl, Phys. Rev. A 93, 069905(E) (2016).

[64] T. Shi, Y.-H. Wu, A. González-Tudela, and J. I. Cirac, Phys. Rev. X 6, 021027 (2016).

[65] T. Shi, Y.-H. Wu, A. González-Tudela, and J. I. Cirac, New J. Phys. 20, 105005 (2018).

[66] M. Bello, G. Platero, J. I. Cirac, and A. González-Tudela, Sci. Adv. 5, eaaw0297 (2019).

[67] J. S. Douglas, H. Habibian, C.-L. Hung, A. Gorshkov, H. J. Kimble, and D. E. Chang, Nat. Photonics 9, 326 (2015).

[68] A. González-Tudela, C.-L. Hung, D. E. Chang, J. I. Cirac, and H. Kimble, Nat. Photonics 9, 320 (2015).

[69] I. de Vega, D. Porras, and J. Ignacio Cirac, Phys. Rev. Lett. 101, 260404 (2008). 
[70] C. Navarrete-Benlloch, I. de Vega, D. Porras, and J. I. Cirac, New J. Phys. 13, 023024 (2011).

[71] L. Krinner, M. Stewart, A. Pazmino, J. Kwon, and D. Schneble, Nature (London) 559, 589 (2018).

[72] M. Aidelsburger, M. Atala, M. Lohse, J. T. Barreiro, B. Paredes, and I. Bloch, Phys. Rev. Lett. 111, 185301 (2013).

[73] O. Astafiev, A. M. Zagoskin, A. A. Abdumalikov, Jr., Y. A. Pashkin, T. Yamamoto, K. Inomata, Y. Nakamura, and J. S. Tsai, Science 327, 840 (2010).

[74] I.-C. Hoi, C. M. Wilson, G. Johansson, T. Palomaki, B. Peropadre, and P. Delsing, Phys. Rev. Lett. 107, 073601 (2011).

[75] I.-C. Hoi, A. F. Kockum, T. Palomaki, T. M. Stace, B. Fan, L. Tornberg, S. R. Sathyamoorthy, G. Johansson, P. Delsing, and C. M. Wilson, Phys. Rev. Lett. 111, 053601 (2013).

[76] A. F. van Loo, A. Fedorov, K. Lalumiére, B. C. Sanders, A. Blais, and A. Wallraff, Science 342, 1494 (2013).

[77] I.-C. Hoi, C. M. Wilson, G. Johansson, J. Lindkvist, B. Peropadre, T. Palomaki, and P. Delsing, New J. Phys. 15, 025011 (2013).

[78] X. Gu, A. F. Kockum, A. Miranowicz, Y. xi Liu, and F. Nori, Phys. Rep. 718-719, 1 (2017).

[79] G. Wendin, Rep. Prog. Phys. 80, 106001 (2017).

[80] P. Roushan, C. Neill, A. Megrant, Y. Chen, R. Babbush, R. Barends, B. Campbell, Z. Chen, B. Chiaro, A. Dunsworth,
A. Fowler, E. Jeffrey, J. Kelly, E. Lucero, J. Mutus, P. J. J. O’Malley, M. Neeley, C. Quintana, D. Sank, A. Vainsencher, J. Wenner, T. White, E. Kapit, H. Neven, and J. Martinis, Nat. Phys. 13, 146 (2016).

[81] B. Peropadre, J. Lindkvist, I. C. Hoi, C. M. Wilson, J. J. GarcíaRipoll, P. Delsing, and G. Johansson, New J. Phys. 15, 035009 (2013).

[82] Y. Chen, C. Neill, P. Roushan, N. Leung, M. Fang, R. Barends, J. Kelly, B. Campbell, Z. Chen, B. Chiaro, A. Dunsworth, E. Jeffrey, A. Megrant, J. Y. Mutus, P. J. J. O’Malley, C. M. Quintana, D. Sank, A. Vainsencher, J. Wenner, T. C. White, M. R. Geller, A. N. Cleland, and J. M. Martinis, Phys. Rev. Lett. 113, 220502 (2014).

[83] B. Peropadre, D. Zueco, F. Wulschner, F. Deppe, A. Marx, R. Gross, and J. J. García-Ripoll, Phys. Rev. B 87, 134504 (2013).

[84] Y. Liu and A. A. Houck, Nat. Phys. 13, 48 (2017).

[85] M. Mirhosseini, E. Kim, V. S. Ferreira, M. Kalaee, A. Sipahigil, A. J. Keller, and O. Painter, Nat. Commun. 9, 3706 (2018).

[86] P. Forn-Díaz, J. J. García-Ripoll, B. Peropadre, J.-L. Orgiazzi, M. A. Yurtalan, R. Belyansky, C. M. Wilson, and A. Lupascu, Nat. Phys. 13, 39 (2017).

[87] S. Mahmoodian, Phys. Rev. Lett. 123, 133603 (2019). 